\title{
Analysis of sporadic neuroendocrine tumours of the enteropancreatic system by comparative genomic hybridisation
}

\author{
H Tönnies, M R Toliat, C Ramel, U F Pape, H Neitzel, W Berger, B Wiedenmann
}

Universitätsklinikum Charité der Humboldt-Universität zu Berlin, Campus Virchow-Klinikum, Institut für Humangenetik, Augustenburger Platz 1, D-13353 Berlin, Germany

$\mathrm{H}$ Tönnies

C Ramel

H Neitzel

Universitätsklinikum Charité der

Humboldt-Universität zu Berlin, Campus Virchow-Klinikum, Medizinische Klinik mit Schwerpunkt

Hepatologie und

Gastroenterologie, Augustenburger Platz 1, D-13353 Berlin, Germany

M R Toliat

U F Pape

B Wiedenmann

Max-Planck-Institut für Molekulare

Genetik, Ihnestraße 73, D-14195 Berlin,

Germany

M R Toliat

W Berger

Correspondence to:

Professor B Wiedenmann.

bertram.wiedenmann@

charite.de

Accepted for publication 31 October 2000

\begin{abstract}
Background-Chromosomal instability is observed in a wide spectrum of human cancer syndromes. However, to date, little is known of the characteristic genetic changes in sporadic neuroendocrine tumours of the gastroenteropancreatic system.
\end{abstract}

Aims and method-We have studied copy number aberrations (CNAs) in 26 sporadic neuroendocrine tumours of the enteropancreatic system (12 foregut and 14 midgut tumours) by comparative genomic hybridisation (CGH), allowing simultaneous evaluation of the entire tumour genome.

Results-Nearly all tumours (25/26; that is, 96\%) showed chromosomal imbalances, including full chromosomal aneuploidies, losses and gains of chromosome arms, interstitial deletions, and amplifications. Whereas gains of chromosomes 4, 5, and 19 were found in both foregut and midgut tumours, gains of chromosomes $20 q(58 \%), 19(50 \%)$, as well as 17 p $(50 \%)$, and partial losses of chromosomes 1p $(42 \%), 2 q(42 \%), 3 p, 4 q$, and $6 q$ (25\% each) were frequently observed only in foregut tumours. In contrast, midgut tumours displayed less CNAs. Gains were detected for chromosomes $17 q$ and $19 p(57 \%)$. Most frequent losses affected chromosomes 18 $(43 \%)$ and $9 p(21 \%)$.

Conclusions-The results of our CGH analyses revealed new distinct candidate regions in the human genome associated with sporadic neuroendocrine tumours. Some of the genetic alterations were shared by foregut and midgut tumours while others discriminated between the two groups. Thus our results allude to the involvement of identical as well as discriminative genetic loci in tumorigenesis and progression of neuroendocrine neoplasms of the foregut and midgut. Based on these findings potential new candidate genes will be discussed.

(Gut 2001;48:536-541)

Keywords: gastroenteropancreatic tumours; comparative genomic hybridisation; foregut; midgut

Malignancies of various tissues and cell types are caused and accompanied by genetic instability, including losses and gains of entire chromosomes or particular fragments. To date, little is known of the genetic alterations in neuroendocrine tumours (NET) of the enteropancreatic system. NETs are rare tumours and occur with an incidence of 1-2/100 000, either sporadically or in association with a rare familial tumour syndrome, designated multiple endocrine neoplasia type 1 (MEN1). The syndrome is characterised by tumours of the parathyroid glands and the endocrine pancreas or duodenum, as well as pituitary tumours. However, tumours in MEN1 patients may also occur at other locations-almost exclusivelyattributable to the foregut, including lung, stomach, and thymus.

According to their location, NETs can be classified into one of three groups: tumours of the foregut (lung, stomach, pancreas, and duodenum), midgut (jejunum, caecum, and ileum), and hindgut (colon and rectum). ${ }^{1}$ Approximately $50 \%$ of NETs present with a clinical syndrome of either hormone or peptide hypersecretion; these tumours are termed functional NETs. However, even in the absence of clinical functionality these tumours synthesise and store secretory products and vesicles, as demonstrated by immunohistochemical staining for the secretory marker molecules synaptophysin and chromogranin A. This in turn indicates that the clinically observed disturbances of hormone, peptide, or neurotransmitter synthesis, storage, and secretion may translate into distinct alterations of genes controlling these pathways. ${ }^{2}$ Malignant behaviour varies widely and depends on size and location of the primary invasive pattern with respect to regional lymph nodes and distant metastasis, cellular atypia of the tumour cells, mitosis, and immunohistochemical staining for the proliferation associated marker Ki67. ${ }^{34}$ Thus depending on the location of the primary tumour, the five year survival rate varies from $65 \%$ for jejunal and ileal NETs to $34 \%$ for pancreatic NETs. ${ }^{5}$

The MEN1 gene, a putative tumour suppressor gene located at $11 \mathrm{q} 13$, was isolated by positional cloning and a large number of mutations have been reported. ${ }^{6}{ }^{7} M E N 1$ encodes a protein which interacts with JUN-D, a transcription factor of the AP1 family, thereby repressing JUN-D mediated transcription activation. ${ }^{8}$ The interaction with JUN-D is abolished by missense mutations detected in the

Abbreviations used in this paper: $\mathrm{CGH}$ comparative genomic hybridisation; CNA, copy number aberration; DAPI, 4',6-diamino-2phenylindole dihydrochloride; NET, neuroendocrine tumours; MEN1, multiple endocrine neoplasia type 1 . 
MEN1 gene of patients. Whereas MEN1 mutations have been identified in more than $90 \%$ of all familial cases, MEN1 mutations are only observed in up to $30 \%$ of all sporadic NETs of the foregut. ${ }^{9}$ By contrast, MEN1 mutations are rarely found in midgut and hindgut tumours. This suggests that other, as yet unidentified, genes must be involved in the tumorigenesis of the foregut as well as the midgut tumours.

To identify additional loci in the human genome which may be implicated in the tumorigenesis of NETs, we have carried out a comparative genomic hybridisation (CGH) survey in 26 tumours (12 foregut and 14 midgut). CGH allows identification of chromosomal regions showing (i) increased copy number (gain or amplification), which may indicate involvement of proto-oncogenes; or (ii) decrease in copy number (deletion of chromosomal material) in regions containing putative tumour suppressor genes. ${ }^{10}$

Distinct chromosomal imbalances were identified in NETs of the foregut and midgut. Based on these findings more detailed molecular analysis of the respective chromosomal intervals are currently under investigation. Candidate genes, potentially involved in the tumorigenesis of NETs, will be presented and discussed.

\section{Patients and methods}

CLINICAL DATA

Tissue samples from 26 patients with histopathologically proved tumour disease were studied. Mean age of the patients was 57.2 years (range 15-72) and there were 10 females and 16 males. Patient data are summarised in table 1. Patients were suffering from neuroendocrine tumours of the gastrointestinal system
(25) or bronchial tract (1). Neuroendocrine tumours were classified as foregut (pancreas nine, duodenum two, bronchial one) or midgut (jejunum one, ileum nine, ileocecal valve three, caecum one) depending on the anatomical location of the primary tumour, according to Williams and Sandler. ${ }^{9}$ This classification was complemented by a tumour biological approach considering immunohistological staining for the neuroendocrine marker molecules synaptophysin, chromogranin A, neurone specific enolase, and the proliferation associated marker Ki67, in addition to location, metastases, and size of the primary tumour. ${ }^{3}$ Metastases had occurred in 21 of 26 neuroendocrine tumours (liver 20, lymph nodes six, ovary one, lung one, pleura one). Hormone or peptide hypersecretion causing a clinical syndrome (that is, functionality) was observed in 19 of 26 neuroendocrine tumours; functional syndromes consisted of the carcinoid syndrome (13), Whipple's triad with insulinoma (three), and Zollinger-Ellison's syndrome and secretory diarrhoea with gastrinoma (three). Tissue samples were collected before patients received any treatment other than surgery.

\section{TISSUE SAMPLES}

All fresh tumour samples, obtained during surgery, were immediately snap frozen. An aliquot was stored at $-70^{\circ} \mathrm{C}$, and the remaining samples were submitted for histological analysis. All tissue samples were microdissected for tumour tissue under direct light microscopic guidance. Reference DNA for CGH analysis was either extracted from peripheral blood from a karyotypically normal male or from the tumour patient investigated (see table 1).

Table 1 Clinical, histopathological data, and comparative genomic hybridisation (CGH) findings of the 26 sporadic neuroendocrine gastroenteropancreatic tumours and six adenocarcinomas

\begin{tabular}{|c|c|c|c|c|c|c|c|}
\hline Case $^{a}$ & Sex/age & Histology & Primary & Metastases & Functionality & CGH gains & CGH losses \\
\hline 1 & $\mathrm{M} / 43$ & NET & Foregut (pancreas, ${ }^{\text {b }}$ gastrinoma) & Liver & + & $15 ; 16 \mathrm{p} ; 17 \mathrm{p} ; 20 \mathrm{q}$ & None \\
\hline 2 & $\mathrm{M} / 34$ & NET & Foregut (pancreas, insulinoma) & No & + & $4 ; 5 ; 11 ; 12 ; 15 ; 18 \mathrm{p} ; 18 \mathrm{q} ; 19 ; 20 ; 21$ & $9 \mathrm{q} ; 22$ \\
\hline 3 & $\mathrm{~F} / 15$ & NET & $\begin{array}{l}\text { Foregut (duodenum/jejunum, } \\
\text { gastrinoma) }\end{array}$ & Liver, lymphnodes & + & None & $3 p ; 20 p$ \\
\hline 4 & $\mathrm{M} / 52$ & NET & Foregut (pancreas, gastrinoma) & Liver & + & $2 \mathrm{q} ; 12 ; 14$ & $3 ; 5 \mathrm{q}$ \\
\hline 5 & $\mathrm{~F} / 69$ & NET & Foregut (pancreas) & Liver & + & $\begin{array}{l}4 ; 5 ; 7 ; 9 ; 12 ; 13 ; 14 ; 16 \mathrm{p} ; 17 ; 18 ; 19 ; 20 \\
21 \mathrm{q} ; 22\end{array}$ & $1 \mathrm{p} ; 2 \mathrm{p} ; 2 \mathrm{q} ; 3 ; 6 \mathrm{q} ; 8 \mathrm{q}$ \\
\hline 6 & $\mathrm{~F} / 67$ & NET & Foregut (pancreas, insulinoma) & No & + & $3 \mathrm{p} ; 5 \mathrm{q} ; 7 ; 10 \mathrm{p} ; 15 \mathrm{q} ; 17 \mathrm{p} ; 19 ; 20 \mathrm{q}$ & $2 \mathrm{q} ; 4 \mathrm{q} ; 12 \mathrm{q} ; 13 \mathrm{q}$ \\
\hline 7 & $\mathrm{M} / 53$ & NET & Foregut (pancreas, insulinoma) & No & + & $8 \mathrm{q} ; 9 \mathrm{p} ; 9 \mathrm{q} ; 10 \mathrm{q} ; 13 \mathrm{q} ; 15 \mathrm{q} ; 21 \mathrm{q}$ & $1 \mathrm{p} ; 1 \mathrm{q} ; 2 \mathrm{q} ; 6 \mathrm{q}$ \\
\hline 8 & $M / 65$ & NET & Foregut (bronchial system) & Liver, pleura & + & $4 ; 7 ; 9 ; 16 ; 19 ; 20 \mathrm{q} ; 22 \mathrm{q}$ & $\begin{array}{l}1 \mathrm{p} ; 2 \mathrm{q} ; 11 \mathrm{p} ; 11 \mathrm{q} ; 12 \mathrm{p} \\
13 \mathrm{q} ; 14 \mathrm{q}\end{array}$ \\
\hline 9 & $M / 49$ & NET & Foregut (pancreas) & Liver, lymphnodes & - & $4 ; 5 ; 7 ; 9 \mathrm{p} ; 12 ; 13 ; 14 ; 17 ; 18 ; 19 ; 20$ & $1 \mathrm{p}$ \\
\hline 10 & $\mathrm{~F} / 65$ & NET & Foregut (duodenum) & No & - & $10 \mathrm{p} ; 11 \mathrm{q} ; 16 \mathrm{p} ; 17 \mathrm{p} ; 17 \mathrm{q} ; 19 ; 20 \mathrm{q} ; 22$ & $18 \mathrm{q}$ \\
\hline 11 & $\mathrm{M} / 60$ & NET & Foregut (pancreas) & Liver, lung & - & $2 \mathrm{p} ; 10 \mathrm{q} ; 20 \mathrm{p}$ & $1 \mathrm{p} ; 1 \mathrm{q} ; 4 \mathrm{q} ; 6 \mathrm{q} ; 11 ; 18$ \\
\hline 12 & $\mathrm{~F} / 37$ & NET & Foregut (pancreas) & Liver & - & $\begin{array}{l}1 \mathrm{q} ; 8 \mathrm{q} ; 11 \mathrm{p} ; 11 \mathrm{q} ; 12 \mathrm{p} ; 12 \mathrm{q} ; 14 \mathrm{q} ; 17 \mathrm{p} ; 17 \mathrm{q} \\
18 \mathrm{q} ; 20 \mathrm{p}\end{array}$ & $2 \mathrm{q} ; 4 \mathrm{q} ; 6 \mathrm{p} ; 7 \mathrm{q} ; 10 \mathrm{p}$ \\
\hline 13 & $\mathrm{~F} / 64$ & NET & Midgut (ileum) & Liver, ovary & + & $4 ; 5 ; 7 ; 9 q ; 10 q ; 14 q ; 17 p ; 17 q ; 19$ & $11 \mathrm{q}$ \\
\hline 14 & $\mathrm{M} / 72$ & NET & Midgut (ileum) & Liver, lymphnodes & + & $4 ; 5 ; 7 ; 14 ; 17 \mathrm{q} ; 20$ & $2 \mathrm{q} ; 3 \mathrm{q} ; 9 ; 13 \mathrm{q} ; 18$ \\
\hline 15 & $\mathrm{M} / 68$ & NET & Midgut (ileum) & Liver, lymphnodes & + & $\begin{array}{l}4 ; 8 \mathrm{q} ; 10 \mathrm{p} ; 10 \mathrm{q} ; 11 \mathrm{q} ; 16 \mathrm{p} ; 17 \mathrm{q} ; 19 ; 20 ; \\
22 \mathrm{q}\end{array}$ & $\begin{array}{l}1 \mathrm{p} ; 2 \mathrm{p} ; 2 \mathrm{q} ; 3 \mathrm{p} ; 6 \mathrm{q} ; 9 \mathrm{p} \\
11 \mathrm{p} ; 18 \mathrm{q}\end{array}$ \\
\hline 16 & $\mathrm{M} / 62$ & NET & Midgut (ileocecal valve) & Liver, lymphnodes & + & None & $18 \mathrm{p}$ \\
\hline 17 & $\mathrm{M} / 59$ & NET & Midgut (caecum) & Lymph nodes & + & $4 ; 10 \mathrm{q} ; 14 ; 17 \mathrm{q} ; 19 \mathrm{p} ; 19 \mathrm{q} ; 20$ & 18 \\
\hline 18 & $\mathrm{~F} / 72$ & NET & Midgut (ileocecal valve) & Liver & + & $4 ; 5 ; 17 \mathrm{q} ; 20 \mathrm{q}$ & None \\
\hline 19 & $\mathrm{~F} / 54$ & NET & Midgut (ileum) & Liver & + & $4 ; 5 ; 13 \mathrm{q} ; 14 \mathrm{q} ; 20 \mathrm{q}$ & $8 \mathrm{p} ; 16 \mathrm{p} ; 17 ; 19 ; 22$ \\
\hline 20 & $\mathrm{~F} / 57$ & NET & Midgut (ileum) & Liver & + & $4 \mathrm{q} ; 5 \mathrm{p} ; 5 \mathrm{q} ; 10 ; 12 \mathrm{q} ; 17 \mathrm{p} ; 19 \mathrm{q}$ & $8 \mathrm{p} ; 11 \mathrm{q} ; 12 \mathrm{p} ; 13 ; 18$ \\
\hline 21 & $\mathrm{M} / 55$ & NET & Midgut (ileum) & Liver & + & $17 \mathrm{q} ; 19 ; 22 \mathrm{q}$ & None \\
\hline 22 & $\mathrm{M} / 65$ & NET & Midgut (ileum) & Liver & + & $19 ; 22 \mathrm{q}$ & $3 ; 9 ; 18$ \\
\hline 23 & $\mathrm{~F} / 59$ & NET & Midgut (ileocecal valve) & Liver & + & $17 \mathrm{q} ; 19 \mathrm{p}$ & 18 \\
\hline 24 & $\mathrm{M} / 69$ & NET & Midgut (jejunum) & Liver & - & $17 \mathrm{q} ; 19 \mathrm{p}$ & None \\
\hline 25 & $\mathrm{M} / 58$ & NET & Midgut (ileum) & No & - & None & None \\
\hline 26 & $\mathrm{M} / 65$ & NET & Midgut (ileum) & Liver & - & $1 \mathrm{p} ; 16 \mathrm{p} ; 19 ; 22$ & None \\
\hline
\end{tabular}

${ }^{\mathrm{a}} \mathrm{CGH}$ analysis was performed with reference DNA of peripheral blood lymphocytes of patients in bold type.

${ }^{\mathrm{b}} \mathrm{CGH}$ analysis was performed on tissue samples highlighted in bold. 

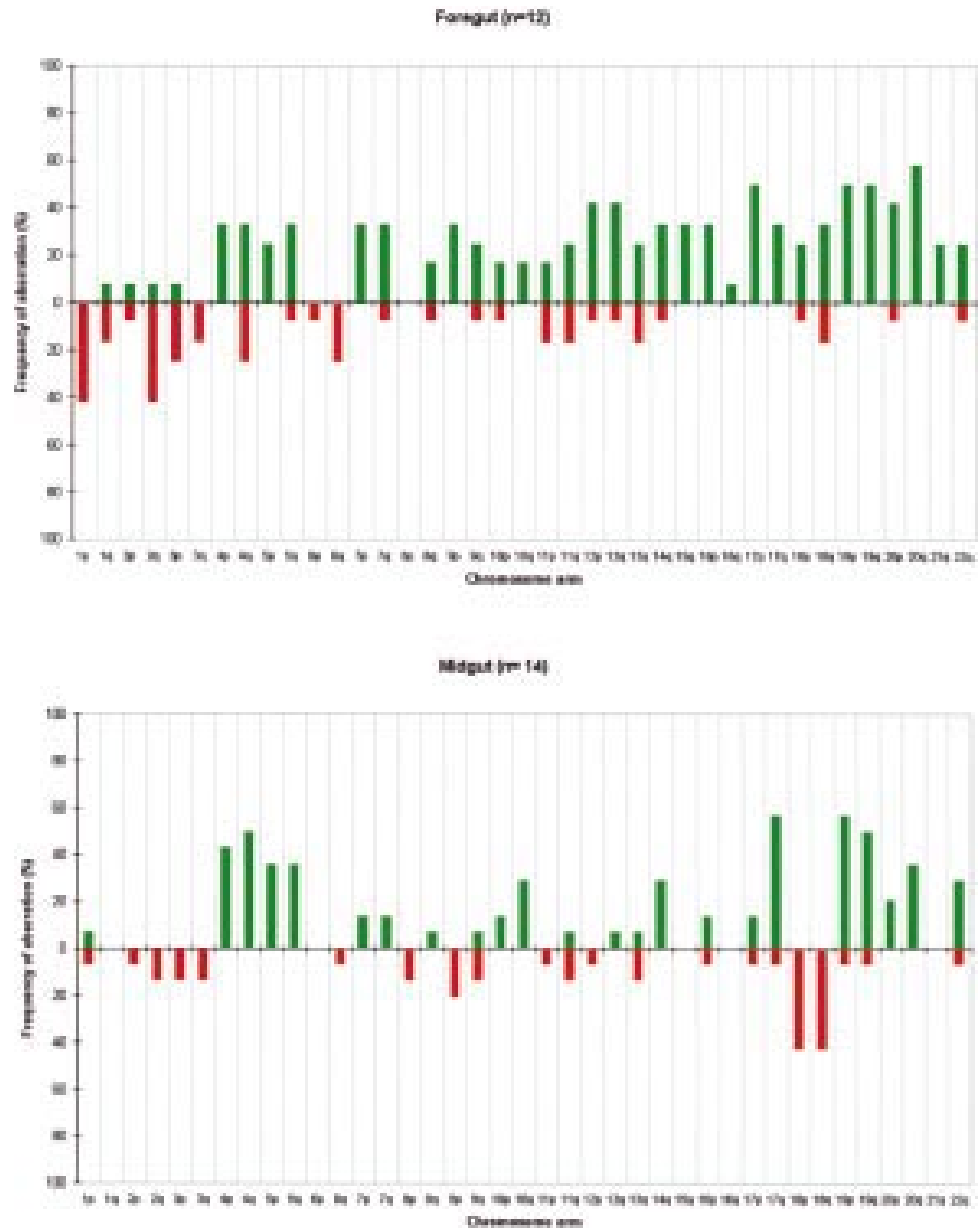

Figure 1 Copy number abnormalities in relation to chromosome arm affected for each tumour group investigated. Gains of chromosomal material are marked by a vertical bar above baseline ( 0 value) and losses below the baseline.

COMPARATIVE GENOMIC HYBRIDISATION

The CGH technique was performed as described previously ${ }^{11}$ with slight modifications. Briefly, high molecular weight genomic DNA was prepared from EDTA blood (reference DNA) and tumour samples (test DNA) according to standard protocols. High molecular weight DNA was labelled by nick translation using direct SpectrumGreen (test DNA) and SpectrumOrange (reference DNA) conjugated dUTP (Vysis). DNase and DNA polymerase I concentrations were chosen to produce labelled probe fragments of 500-1500 base pairs, as observed on a non-denaturing $1.3 \%$ agarose gel.

Metaphase spreads were prepared from PHA stimulated peripheral blood lymphocytes from a karyotypically normal healthy male. Slides were dehydrated in $70 \%, 80 \%$, and $95 \%$ ethanol, denatured for two minutes in $70 \%$ formamide $/ 2 \times \mathrm{SSC}, \mathrm{pH} 7$, at $70-72^{\circ} \mathrm{C}$, followed by further dehydration in ice cold ethanol. For each hybridisation, $400 \mathrm{ng}$ of labelled test DNA, $400 \mathrm{ng}$ of reference DNA, and $50 \mu \mathrm{g}$ of Cot- 1 DNA were mixed and ethanol precipitated. DNA was resuspended in $14 \mu \mathrm{l}$ of hybridisation mix containing $50 \%$ formamide, $2 \times$ SSC, and $10 \%$ dextran sulphate, denatured at $70^{\circ} \mathrm{C}$ for five minutes and applied after 45 minutes preannealing to denatured metaphase spreads. Slides were incubated at $37^{\circ} \mathrm{C}$ in a moist chamber for $2-3$ days. Posthybridisation washes were performed as described previously. ${ }^{12}$ The slides were counterstained with 4',6-diamino-2-phenylindole dihydrochloride (DAPI) and mounted with antifading solution (Vectashield, Vector Laboratories, Inc. Burlingame, California, USA).

\section{DIGITAL IMAGE ANALYSIS}

CGHs were analysed using an epifluorescence microscope (Axiophot, Zeiss, Germany) fitted with different single band pass filter sets for DAPI (blue), Spectrum Green (green), and Spectrum Orange (red) fluorescence. The microscope was equipped with an integrated high sensitivity monochrome charge coupled device camera (Hamamatsu) for image acquisition. Image analysis and karyotyping was performed with an ISIS digital image analysis system (Metasystems, Altlussheim, Germany). Three images (red, green, and blue) were captured from 5-10 metaphases for each tumour sample. Selection and analysis of metaphase spreads were carried out as described previously. ${ }^{13}$ The metaphases were karyotyped and analysed to detect chromosomal aneuploidies on each chromosome. To determine the quality of our CGH approach and to test the diagnostic thresholds, experiments with positive and negative controls were done. As a positive control for CGH, DNA of a bladder carcinoma cell line (EJ30) with well known aberrations was used..$^{14}$ Normal DNAs of probands investigated previously by conventional cytogenetics were used as negative controls showing no ratio deviations crossing our diagnostic thresholds of 0.85 and 1.17 used for the identification of chromosomal under representations (deletions) and over representations (duplications). ${ }^{15}$ The sex chromosomes were excluded from $\mathrm{CGH}$ analysis because hybridisation of test DNAs from different genders would give false signals reflecting the differences in the number of the $\mathrm{X}$ chromosomes in the sample.

\section{Results}

DNA extracted from microdissected shock frozen tumour specimens derived from neuroendocrine tumours of the foregut and midgut was analysed by CGH. Similar to other tumour entities, chromosomal instability was found in all three groups. Foregut and midgut NETs showed common as well as distinct copy number aberrations (CNAs).

\section{FOREGUT TUMOURS}

CNAs were detected in all 12 foregut tumours (mean 13.5 affected chromosome arms/ tumour; range 2-30) (table 1). Gains (72.8\% of aberrations) were more frequent than losses $(27.2 \%)$. The most common chromosomal region for CNAs, found in $58 \%$ of foregut tumours, was gain of $20 \mathrm{q}$ followed by gains of chromosome $19(50 \%)$ and chromosome $17 \mathrm{p}$ $(50 \%$ ) (figs 1, 2). Common chromosome arms for the most frequent partial losses were $1 \mathrm{p}$ (42\%) (common region $1 \mathrm{p} 13-31), 2 \mathrm{q}(42 \%)$ (common region $2 \mathrm{q} 22-32$ ), $3 \mathrm{p}, 4 \mathrm{q}$, and $6 \mathrm{q}$ (25\% each). 


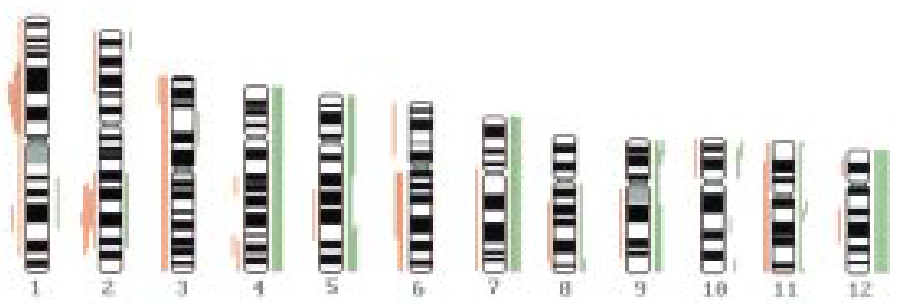

A
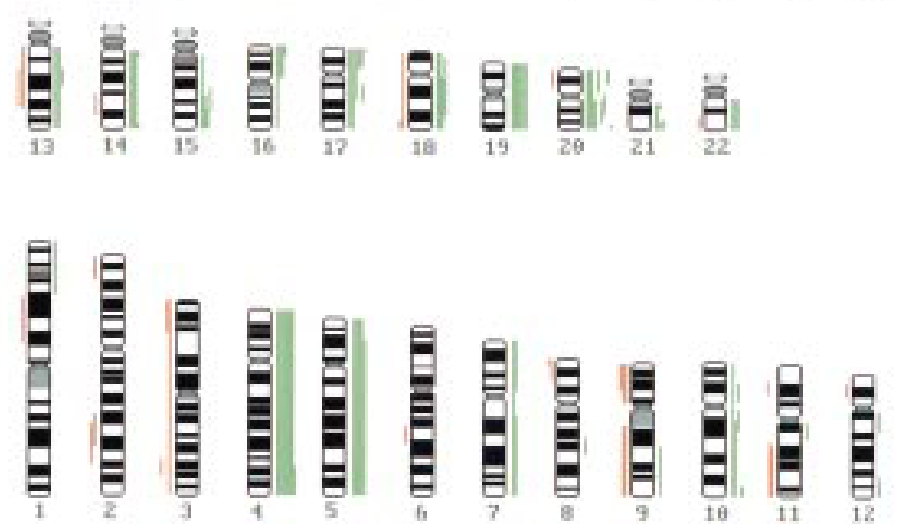

B

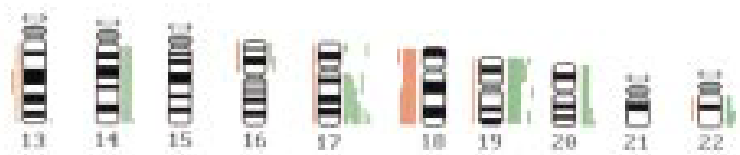

Figure 2 Summary of gains and losses of DNA sequence copy number detected in foregut tumours $(A)$ and midgut tumours (B). Vertical lines on the left side (red) of each

chromosome ideogram represent loss of genetic material in a given tumour at the marked chromosomal segment whereas vertical lines on the right side (green) correspond to gains of genetic material.

Gains of chromosome 4 were associated with gains of chromosome 19 in $36 \%$ of foregut tumours (case Nos 2, 5, 8, 9) (table 1). Deletions in chromosomes $2 \mathrm{q}$ (case Nos 5,6 , 7,12 ) (table 1) and 6q (case Nos 5, 7, 11)
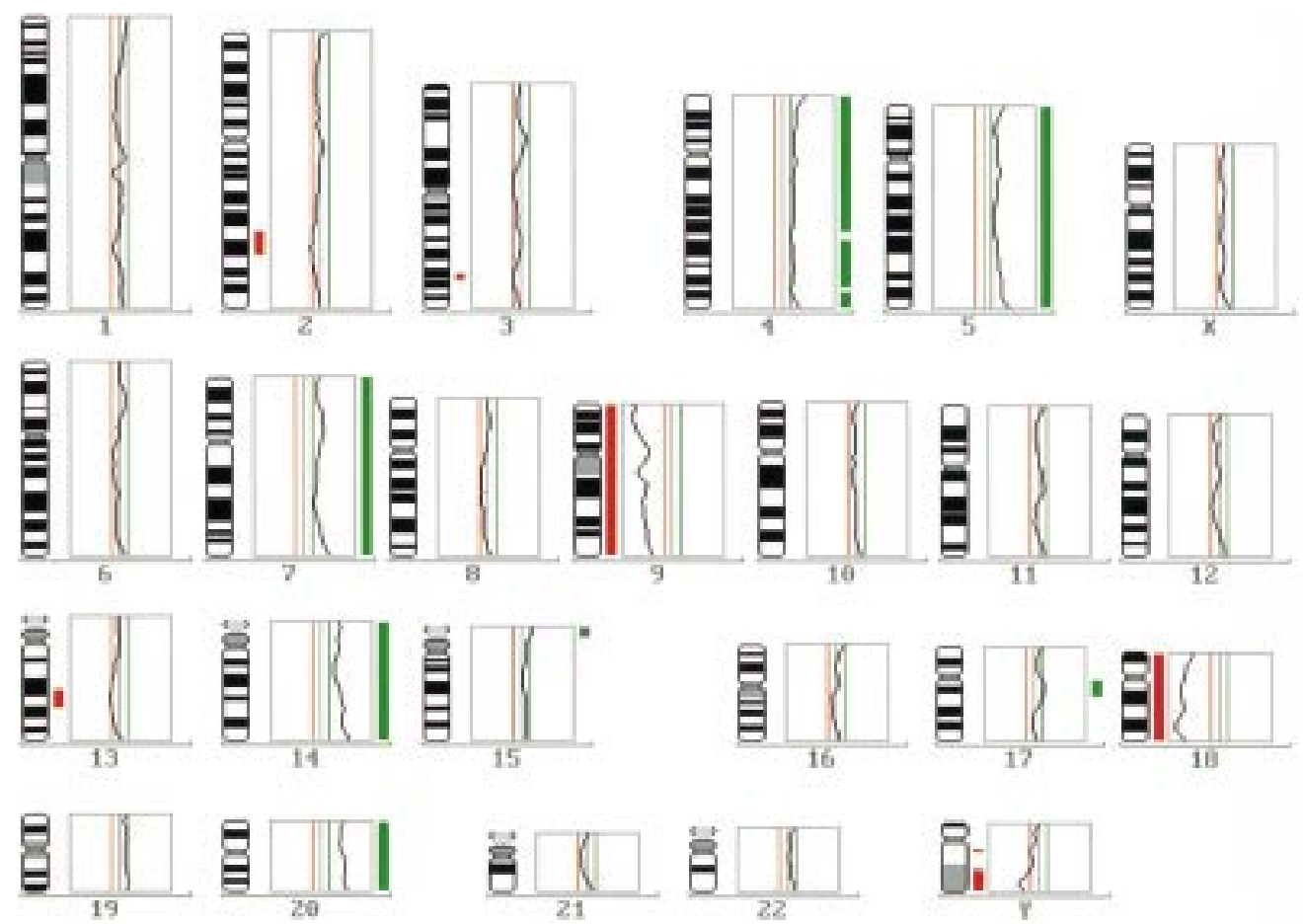

Figure 3 Average ratio profile of tumour 14 (midgut tumour, see table 1). Ratio profiles along the individual chromosomes are shown on the right side of each ideogram. Left (red), middle (grey), and right (green) vertical lanes represent the lower, middle, and upper threshold of the normal range (see text for details). 
was found in $57 \%$ of midgut tumours but in only $27 \%$ of foregut tumours. Similarly, the gain of $20 \mathrm{q}$ was detected in $58 \%$ of foregut tumours versus $36 \%$ in midgut tumours.

The most characteristic differences between foregut and midgut tumours were observed for chromosomes 1, 2, and 18. The frequently observed loss in $1 \mathrm{p}$ and $2 \mathrm{q}$ in foregut tumours $(42 \%)$ was hardly detected in midgut tumours. By contrast, loss of chromosome 18 was common in midgut tumours (43\%) but less frequent in foregut tumours $(18 \mathrm{q}, 18 \%)$. All midgut tumours with full monosomy 18 showed functionality.

\section{Discussion}

We have analysed, using comparative genomic hybridisation, 26 cases of sporadic neuroendocrine gastrointestinal tumours (NET) derived from the foregut $(n=12)$ and midgut $(n=14)$. Our study was designed to assess similarities and differences in the genetic constitution of these two tumour subclasses and to map novel candidate regions within the human genome which are involved in tumorigenesis and progression. Copy number aberrations (CNAs) were more frequent and more complex in foregut tumours (13.5 affected chromosome arms/tumour) compared with neoplasms of the midgut (8.7 affected chromosome arms/ tumour) (fig 3). Both groups showed gains of chromosomes 4, 5, and 19 while other CNAs were found in either group only. Most prominent in the group of foregut tumours was the gain of chromosomal material from $20 \mathrm{q}$ (58\%) and chromosome $19(50 \%)$ and deletions in $1 \mathrm{p}$ and $2 \mathrm{q}$ ( $42 \%$ each). Tumours of the midgut were characterised by gains of $17 \mathrm{q}$ and $19 \mathrm{p}(57 \%)$ and losses of chromosome 18 $(43 \%)$. Some CNAs were combined in a characteristic manner. In $36 \%$ of foregut tumours, gain of chromosome 4 was associated with gain of chromosome 19. Similarly, in midgut tumours, four of six cases with gain of chromosome 4 also showed gain of chromosome 5 and four cases with gain of chromosome 4 showed full or partial trisomy for chromosome 14 . CNAs were also reported in other CGH analyses of sporadic neuroendocrine tumours of the digestive system, including gains of chromosome arms $5 \mathrm{q}, 7 \mathrm{pq}, 12 \mathrm{q}, 14 \mathrm{q}, 17 \mathrm{pq}$, and $20 \mathrm{q}$ and losses of $3 \mathrm{pq}, 6 \mathrm{pq}, 11 \mathrm{pq}$, and $16 \mathrm{p} .{ }^{16}{ }^{17}$ Some were also observed in our group of tumour samples (gains of 5q, 7pq, 12pq, 14q, $17 \mathrm{pq}$, and $20 \mathrm{q}$ ). However, losses of $1 \mathrm{p} 13-\mathrm{p} 31$ and 2 q22-q32 in more than $40 \%$ of foregut tumours and loss of chromosome arms $18 \mathrm{p}$ and/or $18 \mathrm{q}$ in $42 \%$ of midgut tumours have not been described previously.

To date, little is known of the involvement of particular genes. Mutations in MEN1, a putative tumour suppressor gene located on chromosome $11 \mathrm{q} 13$, were identified in 15$20 \%$ of sporadic NETs of the foregut and midgut. ${ }^{18}{ }^{19}$ In the present study, losses of chromosome 11 or deletions in $11 \mathrm{q}$ were observed in four of 26 NETs (case Nos 8, 11, 13, and 20; see table 1). MEN1 mutation screening was performed in three patients (case Nos 11, 13, and 20) but only one sequence alteration was identified (patient No 11, missense mutation G42S, for reference see Toliat and colleagues ${ }^{18}$ ). These results suggest an additional tumour suppressor gene on chromosome 11 possibly involved in tumorigenesis of NETs. This is supported by LOH studies which identified deletions distal to $M E N 1{ }^{20}$ Another tumour suppressor gene, designated $C D K N 2 A$ and located on the short arm of chromosome 9 (9p21), is homozygously deleted or transcriptionally silenced in more than $90 \%$ of gastrinomas and non-functioning pancreatic neuroendocrine tumours. Homozygous deletions are present in $41 \%$ of these tumours; gene silencing by hypermethylation of the promoter region occurs in $58 \% .{ }^{21}$ In our samples, deletions in 9 p were detected in $20 \%$ of midgut tumours and may involve $C D K N 2 A$. However, deletions in $9 \mathrm{p} 21$ may be present more frequently but escape detection due to the low resolution of $\mathrm{CGH}$ compared with deletion screening with gene specific markers. In addition, the group of midgut tumours was characterised by loss of chromosome 18 $(42 \%)$. This chromosome contains the tumour suppressor genes DPC4/SMAD4 and DCC, both located in $18 \mathrm{q} 21$. Interestingly, mutations in DPC4 were found in $50 \%$ of nonfunctioning neuroendocrine tumours of the foregut. ${ }^{22}$ These results and our CGH analyses indicate that $D P C 4$ may be a good candidate gene for the development of NETs derived from the midgut. In addition, another gene involved in regulated secretion may be located on $18 \mathrm{q}$ as all tumours with a deletion in $18 \mathrm{q}$ demonstrated functionality. Thus $18 \mathrm{q}$ may be a candidate region for a gene that participates in the regulation of secretion by possibly inhibiting secretion unless the appropriate stimulus is elicited. Once this gene is deleted and/or inactivated by point mutations, regulation of the secretory process might be abolished, thus leading to functionality with a clinical syndrome of hypersecretion.

In contrast with the group of midgut tumours (which was characterised by gains of $17 q$ and $19 p$, and losses of chromosome 18), neoplasms attributable to the foregut were dominated by gains of $20 \mathrm{q}$ and 19 and partial losses of chromosome arms $1 \mathrm{p}$ and 2q. Although deletions of the short arm of chromosome 1 (1p32-p33) were frequently observed in an aggressive subtype of endometrial cancer, ${ }^{23}$ no prominent tumour suppressor gene was isolated from the corresponding interval. The high frequency of $1 \mathrm{p}$ deletions $(42 \%)$ in foregut tumours suggests an important gene within this chromosomal region, which initiates tumorigenesis or contributes to the progression of foregut NETs. The same holds true for the long arm of chromosome 2 where deletions were detected with the same frequency $(42 \%)$. This region is also deleted in small cell lung carcinoma. ${ }^{24}$

Chromosome instability is often found in tumour cells and can result from uncontrolled cell proliferation and defective DNA repair systems or may be due to alterations in genes which are directly involved in chromosome segregation. The latter was shown for STK15/ 
$B T A K$ and $B R C A 1$, two genes which are required for normal centrosome number and function. ${ }^{26}{ }^{27}$ Gene amplification or overexpression of $S T K 15$, a centrosome associated kinase, leads to multiple functional centrosomes in a single nucleus. Consequently, chromosomes segregate unequally, resulting in aneuploidy and transformation of cells. The STK15 gene is located in $20 \mathrm{q} 13$, a region which showed gain of chromosomal material in $58 \%$ of foregut tumours. Accordingly, STK15 overexpression or an increased gene dosage may contribute to the genetic instability in neuroendocrine tumours of the foregut.

In conclusion, the $\mathrm{CGH}$ data presented here define a set of genomic regions in the human genome that are likely to harbour genes which play an important role in the genesis and progression of neuroendocrine tumours of the enteropancreatic system. Further molecular genetic studies will identify more precisely the relevant regions and hopefully lead to identification of genes and mutations associated with neuroendocrine malignancies.

The authors are grateful to I Eichhorn and A Gerlach for excellent technical assistance.

1 Williams ED, Sandler M. The classification of carcinoid tumors. Lancet 1963;1:238-9.

2 Wiedenmann B, John M, Ahnert-Hilger G, et al. Molecula and cell biological aspects of neuroendocrine tumors of the gastroenteropancreatic system. $f$ Mol Med 1998;76:637 gastroc.

3 Capella C, Heitz PU, Hofler H, et al. Revised classification of neuroendocrine tumours of the lung, pancreas and gut. Virchows Arch 1995;425:547-60.

4 Rindi G, Capella C, Solcia E. Cell biology, clinicopathological profile, and classification of gastro-enteropancreatic cal profile, and classification of gastro-enteropa
endocrine tumors. $\mathcal{F}$ Mol Med 1998;76:413-20.

5 Modlin IM, Sandor A. An analysis of 8305 cases of carcinoid tumors. Cancer 1997;79:813-29.

6 Chandrasekharappa SC, Guru SC, Manickam P, et al. Positional cloning of the gene for multiple endocrine neoplasiatype 1. Science 1997;276:404-7.

7 Lemmens I, Van de Ven WJ, Kas K, et al. Identification of the multiple endocrine neoplasia type 1 (MEN1) gene. The European Consortium on MEN1. Hum Mol Genet 1997;6: $1177-83$

8 Agarwal SK, Guru SC, Heppner C, et al. Menin interacts with the AP1 transcription factor JunD and represses JunD-activated transcription. Cell 1999;96:143-52.

9 Debelenko LV, Brambilla E, Agarwal SK, et al. Identification of MEN1 gene mutations in sporadic carcinoid tumors of the lung. Hum Mol Genet 1997;6:2285-90.
10 Kallioniemi A, Kallioniemi OP, Sudar D, et al. Comparative Kallioniemi A, Kallioniemi OP, Sudar D, et al. Comparative genomic hybridization for molecular cyt

11 Clarke DJ, Gimenez-Abian JF, Tonnies H, et al. Creation of monosomic derivatives of human cultured cell lines. Proc Natl Acad Sci USA 1998;95:167-71.

12 Kallioniemi OP, Kallioniemi A, Piper J, et al. Optimizing comparative genomic hybridization for analysis of DNA sequence copy number changes in solid tumors. Genes Chromos Cancer 1994;10:231-43.

13 du Manoir S, Schröck E, Bentz M, et al. Quantitative analysis of comparative genomic hybridization. Cytometry 1995; 19:27-41.

14 Richter J, Jiang F, Görög J-P, et al. Marked genetic differences between stage pTa and stage pT1 papillary bladder cancer detected by comparative genomic hybridization. Cancer Res 1997;57:2860-4.

15 Larramendy ML, El-Rifai W, Knuutila S. Comparison of fluorescein isothiocyanate- and Texas red-conjugated nucleotides for direct labeling in comparative genomic hybridization. Cytometry 1998;31:174-9.

16 Terris B, Meddeb M, Marchio A, et al. Comparative genomic hybridization analysis of sporadic neuroendocrine tumors of the digestive system. Genes Chromos Cancer 1998;22:50-6.

17 Speel EJM, Richter J, Moch H, et al. Genetic differences in endocrine pancreatic tumor subtypes detected by comparative genomic hybridization. Am $\mathcal{F}$ Pathol 1999;155:1787tive 94.

18 Toliat MR, Berger W, Ropers H-H, et al. Mutations in the $M E N I$ gene in sporadic neuroendocrine tumors of the gastroenteropancreatic system. Lancet 1997;350:1223.

19 Görtz B, Roth J, Krähenmann A, et al. Mutations and allelic deletions in the $M E N 1$ gene are associated with a subset of sporadic endocrine pancreatic and neuroendocrine tumors and not restricted to foregut neoplasms. Am F Pathol 1999; 154:429-36.

20 Chakrabarti R, Srivatsan ES, Wood TF, et al. Deletion mapping of endocrine tumors localizes a second tumor suppressor gene on chromosome band 11q13. Genes Chromos Cancer 1998;22:130-7.

21 Muscarella P, Melvin WS, Fisher WE, et al. Genetic alterations in gastrinomas and nonfunctional pancreatic neuroendocrine tumors: an analysis of p16/MTS1 tumor suppressor gene inactivation. Cancer Res 1998;58:237-40.

22 Bartsch D, Hahn SA, Danichesski KD, et al. Mutations of the DPC4/Smad4 gene in neuroendocrine pancreatic tumors. Oncogene 1999;18:2367-71.

23 Arlt MF, Herzog TJ, Mutch DG, et al. Frequent deletion of chromosome $1 \mathrm{p}$ sequences in an aggressive histologic subtype of endometrial cancer. Hum Mol Genet 1996;5:101721 .

24 Kohno T, Morishita K, Takano H, et al. Homozygous deletion at chromosome 2 q33 in human small-cell lung tion at chromosome $2 \mathrm{q} 33$ in human small-cell lung carcinoma identified by arbitrarily primec

25 Kohno T, Otsuka T, Takano H, et al. Identification of a novel phospholipase C family gene at chromosome $2 \mathrm{q} 33$ that is homozygously deleted in human small cell lung carcinoma. Hum Mol Genet 1995;4:667-74.

26 Zhou H, Kuang J, Zhong L, et al. Tumour amplified kinase STK15/BTAK induces centrosome amplification, aneuploidy and transformation. Nat Genet 1998;20:189-93.

$27 \mathrm{Xu} \mathrm{X}$, Weaver Z, Linke SP, et al. Centrosome amplification and a defective G2-M cell cycle checkpoint induce genetic instability in BRCA1 exon 11 isoform-deficient cells. Mol Cell 1999;3:389-95. 